Théologiques

Théologiques

\title{
Le monde du travail et de l'emploi au Mexique
}

\section{Carlos Heredia}

Volume 3, numéro 2, octobre 1995

Crise du travail, crise de civilisation

URI : https://id.erudit.org/iderudit/602422ar

DOI : https://doi.org/10.7202/602422ar

Aller au sommaire du numéro

\section{Éditeur(s)}

Faculté de théologie de l'Université de Montréal

\section{ISSN}

1188-7109 (imprimé)

1492-1413 (numérique)

Découvrir la revue

\section{Citer cet article}

Heredia, C. (1995). Le monde du travail et de l'emploi au Mexique.

Théologiques, 3(2), 33-44. https://doi.org/10.7202/602422ar

\section{Résumé de l'article}

Le processus de mondialisation de l'économie a eu un impact très négatif sur le travail et sur l'emploi au Mexique. Le programme d'ajustement structurel mis de l'avant par la Banque mondiale, l'entrée du Mexique au sein du GATT (Accord général des tarifs douaniers et du commerce) en 1986, et de façon encore plus accélérée, l'entrée en vigueur du Traité de libre-échange nord-américain ont favorisé la mise en oeuvre d'un faux concept de compétitivité qui repose sur la dévalorisation du travail. Sans une démocratisation de l'économie et de la politique, sa revalorisation sera impossible. 


\title{
Le monde du travail et de l'emploi au Mexique ${ }^{1}$
}

\author{
Carlos HEREDIA \\ Économiste et directeur de programme \\ Equipo Pueblo2, Mexico
}

\section{RÉSUMÉ}

Le processus de mondialisation de l'économie a eu un impact très négatif sur le travail et sur l'emploi au Mexique. Le programme d'ajustement structurel mis de l'avant par la Banque mondiale, l'entrée du Mexique au sein du GATT (Accord général des tarifs douaniers et du commerce) en 1986, et de façon encore plus accélérée, l'entrée en vigueur du Traité de libre-échange nord-américain ont favorisé la mise en cuvre d'un faux concept de compétitivité qui repose sur la dévalorisation du travail. Sans une démoctatisation de l'économie et de la politique, sa revalorisation sera impossible.

Malgré les apparences de démocratie, la présence ininterrompue du même parti au pouvoir depuis 1929 a créé, au Mexique, une situation tout à fait particulière dans tous les secteurs de la vie nationale. C'est le cas de celui du travail dont la dynamique est fortement marquée par le cadre politique, institutionnel et juridique qui a été mis en place au cours des

1 Traduit de l'espagnol par Madeleine Perreault, en collaboration avec Michel Beaudin.

2 N.D.L.R. Equipo Pueblo est une organisation non-gouvernementale composée d'une trentaine de permanents et qui appuie, de façon étroite, les organisations et coalitions sociales indépendantes à travers le Mexique. Il se consacre à la promotion de la démocratie, du développement social et de la justice économique ainsi qu'à la défense des droits humains. Il publie une revue diffusée internationalement : La otra cara de Mexico (L'autre visage du Mexique). Equipo Pueblo a été fondé en 1977 par le Père Alex Morelli, un prêtre œuvrant dans les bidonvilles de Mexico et qui suscita autour de lui l'engagement de jeunes universitaires. 
décennies passées. Cette particularité mexicaine revêt d'autant plus d'importance que l'Accord de libre-échange nord-américain (ALÉNA) livre maintenant les trois économies nationales du continent à une concurrence ouverte. Il m'est donc apparu pertinent de faire d'abord état de ce cadre avant d'aborder la question du travail comme telle et son contexte économique récent.

\section{Le cadre institutionnel et légal du travail au Mexique}

Les syndicats mexicains ont toujours été une importante source d'appui pour le parti politique actuellement au pouvoir (le Parti révolutionnaire institutionnel - PRI), depuis sa fondation en 1929. Les directions syndicales sont en général davantage préoccupées d'être en bons termes avec le gouvernement que de promouvoir les intérêts de leurs membres de la base. Une organisation-parapluie sert de véhicule à la subordination des travailleurs: le Congrès des travailleurs (CT). Le CT est largement dominé par la plus forte organisation, la Confédération des travailleurs du Mexique (CTM), dont le dirigeant, Fidel Velásquez, maintenant âgé de 95 ans, a été l'homme fort du syndicalisme depuis un demi-siècle.

Le Mexique compte une population active d'environ 36 millions de personnes dont près de 10 millions sont syndiquées, ce qui représente un taux de syndicalisation de $28 \%$. La CTM prétend regrouper 4 millions de membres. Des syndicats indépendants ont été formés à gauche (Le Front authentique du travail - FAT) de même qu'à droite, et dans ce dernier cas, principalement par les hommes d'affaires de Monterrey (La Fédération nationale des syndicats indépendants - FNSI), mais ils n'ont, au total, jamais dépassé 1 million d'affiliés. D'autres syndicats officiels mais hors du contrôle du CT complètent ce tableau du monde syndical. Parmi les syndicats nationaux les plus puissants, mentionnons le Syndicat des professeurs (SNTE), le Syndicats des travailleurs du pétrole (STPRM) et le Syndicat des travailleurs du téléphone (STRM).

On fait souvent valoir que la législation mexicaine du travail serait extrêmement avancée. En supposant que ce soit le cas, ce que nous contestons, l'inexistence, en pratique, de l'État de droit transforme les droits des travailleurs en véritable farce. Voilà pourquoi il ne vaut pas la peine de parler d'autres droits que de ceux dont jouissent effectivement les travailleurs, en d'autres termes des droits acquis.

Pour le reste, la législation se réduit à quelques cas précis. Par exemple, chez les fonctionnaires de l'État, la syndicalisation atteint presque $100 \%$ mais le droit de libre affiliation n'est pas respecté. Ainsi, en entrant dans la fonction publique, les travailleurs deviennent automati- 
quement membres de la Fédération des syndicats des travailleurs au service de l'État (FSTSE) qui, dans les faits, constitue une simple ramification du parti officiel. De plus, la législation mexicaine du travail ne permet pas les grèves dans le secteur public, ce qui place ce groupe de travailleurs dans un régime d'exception.

Il existe bien d'autres limites à l'exercice de leurs droits par les travailleurs. Ainsi, en principe, ceux-ci peuvent signaler une injustice dont ils se sentent victimes et présenter une plainte directement au Bureau de la défense du travail. Celui-ci devrait supposément fonctionner comme un ombudsman et un avocat des travailleurs, surtout de ceux qui ont peu de recours économiques. En pratique, toutefois, ce Bureau ne possède aucun pouvoir pour obliger les entreprises à respecter ses recommandations.

L'Assemblée fédérale de conciliation et d'arbitrage, un tribunal qui a pour fonction de résoudre les conflits de travail, utilise des procédures tant individuelles que collectives concernant l'interprétation et l'application des normes du travail. On dispose des cas surtout de façon verbale. L'Assemblée est étroitement contrôlée par le Secrétariat (Ministère) du travail qui gère les conflits de travail en étroite conformité avec des critères politiques. On a en vu récemment un avec le conflit qui a éclaté entre la Ville de Mexico et le Syndicat unique des travailleurs du transport en commun urbain (SUTAUR-100), à partir d'avril 1995. Invoquant une Loi des faillites qui ne peut normalement s'appliquer qu'aux entreprises privées, l'administration de la ville a mis en faillite Ruta-100, une compagnie lui appartenant, pour justifier le congédiement de 12500 travailleurs qui échappaient à son contrôle politique, sous prétexte que le syndicat SUTAUR-100 avait recueilli des fonds pour appuyer l'Armée zapatiste de libération nationale.

En théorie, le droits des travailleurs, garantis par la Constitution, sont appliqués par la Loi fédérale du travail et par la Loi de la sécurité sociale. Sur papier, les normes et la protection légale au Mexique sont, dans certains cas, plus étendues que celles que l'on trouve dans la législation du travail aux États-Unis, par exemple. Dans les faits, cependant, l'application des mesures pratiques, réglementaires ou concernant la protection laisse beaucoup à désirer et est abandonnée au bon vouloir ou à l'arbitraire des fonctionnaires officiels en matière du travail.

Le Mexique a formellement ratifié 74 conventions de l'Organisation internationale du travail (OIT) sur les normes du travail. Ces conventions se transforment automatiquement en lois une fois qu'elles ont été ratifiées même si, dans la pratique, elles sont rarement appliquées. Les travailleurs mexicains du secteur formel sont protégés par l'Institut mexicain de 
la sécurité sociale (IMSS), et ceux du secteur public, par l'Institut de sécurité et de services sociaux pour les travailleurs de l'État (ISSSTE).

Au mois de décembre de chaque année, les employeurs doivent remettre à leurs travailleurs des étrennes pour une valeur équivalant à au moins quinze jours de salaire. Les travailleurs syndiqués reçoivent généralement quatre semaines de salaire à ce titre. Les patrons doivent aussi réserver $10 \%$ de leurs profits pour le partage annuel des bénéfices avec leurs travailleurs. Mais quand elles font des profits, très peu d'entreprises mexicaines respectent cette clause.

Selon la loi, les travailleurs mexicains ne peuvent être congédiés sans raison valable. Cependant, comme le montre bien l'exemple des travailleurs du transport urbain de la Ville de Mexico, le gouvernement luimême peut facilement, pour des motifs politiques, inventer des "raisons valables " de se départir, à sa guise, de travailleurs.

Même si la Constitution mexicaine de 1917 dans l'Article 123 consacre beaucoup d'articles à la protection des droits du travail, en pratique le gouvernement s'immisce systématiquement dans la vie interne des syndicats et veille à la mise en application de la loi du travail seulement quand cela n'entre pas en concurrence avec ses intérêts politiques. Alors, la plupart du temps, des lois qui semblent, sur papier, très favorables aux travailleurs, ne sont tout simplement pas appliquées dans la pratique.

\section{Contrôle politique et milieu de travail}

L'absence de liberté d'organisation pour les syndicats et les obstacles quotidiens rencontrés par les travailleurs pour élire leurs propres dirigeants selon des critères cohérents avec leurs intérêts empêchent tout simplement la création de moyens autonomes d'expression et de participation. La participation des travailleurs reste trop exceptionnelle dans les milieux de travail. En général les gens d'affaires favorisent la subordination des syndicats. Les entreprises aiment à traiter avec des syndicats dociles pour toute négociation menant à l'adoption d'une convention collective. Elles ne se gêneront pas, à l'occasion, pour dire que les syndicats mexicains ont une culture du travail très arriérée, mais ce faisant, elles ne font que mettre le doigt sur un problème qu'elles ont elles-mêmes contribué à créer depuis des années.

Au début du soulèvement de l'Armée zapatiste de libération nationale (EZLN), le $1^{\text {er }}$ janvier 1994, plusieurs secteurs sociaux se sont regroupés sous des organisations-parapluies nationales. Les syndicats indépendants de travailleurs ont mis sur pied la Convention nationale des 
travailleurs (CNT), et la Coordination inter-syndicale "Premier mai " a été formée en 1995. Ce sont là deux exemples des efforts les plus récents pour regrouper ce qu'on a appelé les "courants démocratiques" des syndicats officiels et les syndicats indépendants.

La politique de l'administration Salinas à l'égard du travail - tout comme celle de ses prédécesseurs - était très simple et très claire : faire tout le nécessaire pour s'assurer de l'appui des syndicats (ou du moins de leurs dirigeants) pour la mise en cuvre de la politique gouvernementale d'ajustement économique. Le Secrétaire au Travail, Arsenio Farell, un avocat patronal à la main de fer, a occupé ce poste à deux reprises de 1982 à 1994. Il a adopté une ligne d'action visant au contrôle politique du travail. Durant les onze ans et quatre mois où il a exercé ses fonctions, Farell a pratiquement éliminé les organisations ouvrières indépendantes, divisé ou démantelé les syndicats officiels, imposé des plafonds par le moyen d' "accords » et mis en pratique un style impitoyable de "négociations" grâce auquel il a forcé les syndicats à accepter des politiques du travail toutes décidées à l'avance par le gouvernement .

Les syndicats indépendants ont dû faire face à des difficultés toujours plus grandes pour exercer leurs droits constitutionnels, tel le droit de libre association et le droit de grève. Farell a été tellement efficace qu'il n'a même pas été nécessaire d'amender la loi du travail, comme les industriels mexicains et les investisseurs étrangers le demandaient depuis longtemps, pour rendre plus flexibles les règles d'embauche et de congédiement. Farell a, en fait, rendu la loi inutile et a mis en ouvre une politique à sens unique : il avait le dernier mot dans tout conflit de travail et, plus souvent qu'autrement, le critère adopté pour régler l'affaire était politique et non pas légal.

Rien de surprenant, donc, qu'avec un tel contrôle sur le monde du travail, le gouvernement du Mexique ait rencontré moins de résistance de la part des leaders syndicaux que les gouvernements des autres pays lors de l'implantation de son sévère plan d'austérité. En 1976, le pouvoir d'achat des travailleurs a atteint un sommet historique. Les salaires se sont ensuite mis à baisser pendant quelques années pour remonter en 1981. Depuis 1982, à cause des politiques d'ajustement structurel imposées par la Banque mondiale et le Fonds monétaire international (FMI) et visant l'atteinte de «salaires compétitifs ", la rémunération des travailleurs n'a cessé de dégringoler, perdant environ $67 \%$ de son pouvoir d'achat au cours des treize dernières années, y compris une perte estimée à 23\% pour 1995 . 


\section{Crise économique et restructuration du travail}

Une baisse des salaires réels est devenue une mesure d'ajustement habituelle, au Mexique, quand il s'agit de réaliser une stabilisation macroéconomique et des réformes structurelles. La Banque mondiale recommande, de plus, l'élimination des institutions du marché du travail et des règlements qui "restreignent la mobilité du travail", tels les primes de congédiement élevées et les bénéfices marginaux. Les dirigeants du secteur privé continuent d'essayer de rendre la main-d'œuvre encore plus "flexible " en cherchant à obtenir le droit de signer des contrats où les travailleurs seraient payés uniquement pour les heures travaillées et de réduire encore davantage les avantages sociaux.

Pour ce qui est de l'évolution de l'emploi, le Mexique a été cité comme un modèle de réussite d'un pays où l'ajustement structurel s'est accompagné d'une véritable réduction des salaires afin d'éviter des mises à pied massives ${ }^{3}$. D'après le gouvernement, même après le déclenchement de la crise, le taux de chômage officiel ne dépassait pas $5,6 \%$ en juin 1995. Le Congrès du travail (CT), traditionnellement pro-gouvernemental croit toutefois qu'il s'élèverait plutôt à $20 \%$ de la population économiquement active alors que plus de $40 \%$ de celle-ci serait réduite au sousemploi. Le gouvernement ne tient compte que du chômage urbain et que des chômeurs qui ont droit à des prestations alors que le problème est beaucoup plus grave dans les zones rurales. Malheureusement, le gouvernement ne produit pas de statistiques sur le sous-emploi. D'autre part, parce que le Mexique ne possède pas un système de sécurité sociale efficace, peu de gens ont les moyens de rester au chômage pour une longue période.

Le poids de la crise économique - qui se traduit par des licenciements, par une chute des revenus et par la stagnation des prestations sociales - continue de retomber sur les travailleurs sans qu'on voit apparaître dans le panorama national une option syndicale moderne capable de faire face aux problèmes de cette restructuration économique et de cette transition politique bourrée d'incertitudes avec lesquelles le pays est aux prises.

Dans le cadre des licenciements massifs occasionnés par la crise, les travailleurs congédiés non seulement grossissent les rangs des chômeurs, mais ils perdent pratiquement l'espoir de pouvoir jamais revenir sur le

3 Vittorio CORBO, Stanley FISHER, Adjustement Lending Revisited: Policies to Restore Growth. The World Bank, 1992, p. 11. 
marché du travail. Ils ne peuvent même pas, non plus, aspirer à reprendre leur emploi ou encore à en trouver un autre à court terme.

Il y a plus d'un million de travailleurs qui arrivent sur le marché du travail à chaque année. Entre 1988 et 1994, cependant, seulement 400000 nouveaux emplois ont été créés annuellement dans le secteur "moderne " de l'économie, ce qui a accru de façon significative le déficit de l'emploi et s'est traduit également par un gonflement du secteur informel. Historiquement, le Mexique a toujours connu une économie informelle passablement importante, mais durant les années 80 , ce secteur a pris rapidement de l'extension comme conséquence de la crise économique et de l'ajustement structurel. Selon l'Institut national de statistiques géographiques et d'informatique (INEGI), l'économie informelle, en pourcentage du produit intérieur brut (PIB), est passé d'environ $12,9 \%$ en 1986 à $22,5 \%$ en 1991, et emploie environ le tiers de la force de travail active. D'autres sources estiment que ce pourcentage est encore plus élevé (32\% du PIB et $50 \%$ de la population économiquement active $\left.{ }^{4}\right)$. Les travailleurs de l'économie informelle vont des vendeurs de rue aux employés des maquiladoras ${ }^{5}$ qui travaillent dans de petites entreprises loin de leur foyer. Le revenu mensuel moyen d'un travailleur du secteur informel est estimé à $184 \$$ US, soit environ le double du salaire minimum. Ce secteur a constitué une source de revenus pour des millions de personnes durant la crise économique, réduisant la probabilité d'un soulèvement social.

4 Ursula Oswald, Estratégias de Supervivencia en la Cirudad de México. Universidad Nacional Autónoma de México, Centro Regional de Investigaciones Multidisciplinarias, Cuernavaca, México, 1991, p. 40.

5 Zones franches d'assemblage de produits destinés exclusivement à l'exportation et établies depuis 1966 le long de la frontière nord du Mexique. Les conditions offertes permettent aux entreprises américaines surtout de retrouver leur * compétitivité $»$ : salaire horaire moyen de $1 \$$ US, coûts d'installation très bas, infrastructures et autres services, impôt dérisoire, disponibiliré de main-d'œuvre, contrôle syndical, etc. Les maquiladoras comptaient, en 1991, 1886 entreprises fournissant de l'emploi à 437147 travailleurs et travailleuses (Bertha LUJAN, « Les maquiladoras mexicaines: l'enfer se poursuit ", dans Coalition québécoise sur les négociations trilatérales, ALENA. Les gagnants et les perdants. Montréal, déc. 1992, p. 9.) 


\section{QUELQUES CHIFFRES sur la crise récente et ses coûts sociaux}

- Le 20 décembre 1994, le Mexique est contraint par les institutions financières internationales, en raison de sa crise financière, de dévaluer le peso de $50 \%$ (de 2,5 à 5 pesos pour $1 \$ C D N$ ) et d'adopter un plan d'austérité (9 mars 1995). Depuis, l'industrie nationale et le niveau de vie s'effondrent.

- Les taux d'intérêt ont monté jusqu'à 109\% pour redescendre autour de 50\% à l'été 1995 .

- On s'attend à un taux d'inflation de $50 \%$ en 1995, alors que les hausses salariales sont limitées à $10 \%$.

- 6000 usines auraient fermé leurs portes entre décembre 1994 et août 1995. Pour les firmes transnationales, cependant, la dévaluation du peso a marqué une baisse des coûts de production et facilité les exportations, vers les États-Unis surtout.

- Au cours de la même période, 1200000 personnes auraient perdu leur emploi, selon les chiffres officiels. En réalité, les pertes d'emplois pourraient s'élever à 3 millions.

- Les ventes au détail ont baissé de $25 \%$. L'économie se tourne vers les seules exportations.

\section{Sources :}

B. DE LA GRANCE, "L'économie mexicaine traverse une profonde récession ", Le Devoir, 10 août 1995.

RQIC, La dimension sociale de l'intégration continentale. Montréal, mai 1995. 
Les salaires ont chuté de $36 \%$ à $22 \%$ du PIB durant les années 80 alors que le capital a monté de $54 \%$ à $62 \% 6$. La politique gouvernementale de contrôle des salaires n'a survécu que grâce à un "accord " entre les plus hautes autorités, le milieu des affaires, les paysans et les travailleurs. Sur papier, il s'agit d'un mécanisme de contrôle des prix et des salaires. Dans la pratique, depuis sa mise en vigueur en 1987, l'" Accord " ou "Pacte" a fonctionné comme un mécanisme de gel des salaires pendant qu'il libérait lentement le reste des prix dans l'économie. Le Mexique a maintenant le plus bas salaire minimum des 25 pays membres de l'Organisation de coopération et de développement économiques (OCDE). En juin 1995, le salaire minimum était de 18,4 nouveaux pesos ( $2,95 \$$ US) par jour, soit environ le douzième du salaire minimum aux États-Unis.

Le programme d'ajustement économique du gouvernement du Président Ernesto Zedillo (1994-2000), inauguré le 9 mars 1995 pour faire face à la crise économique qui sévit depuis la dévaluation du 19 décembre 1994, a produit une grande vague de mécontentement social. Les coûts sociaux ${ }^{7}$ provoqués par le plan de choc commencent à se manifester sur le terrain du travail et on commence à entrevoir une profonde crise des alliances politiques entre des syndicats officiels et le gouvernement. La manifestation de la Fête des travailleurs, du $1^{\text {er }}$ mai 1995, alors que plus 600000 personnes protestèrent dans les rues de Mexico contre le programme d'ajustement, a fait ressortir ce mécontentement général, mais elle a aussi révélé l'absence de leadership cohérent au sein du mouvement ouvrier.

\section{La mondialisation économique et l'avenir de l'emploi au Mexique}

L'origine de la crise actuelle au Mexique tient surtout au modèle économique qui a été orchestré à partir de 1982, un modèle extrêmement dépendant des mouvements financiers internationaux. Ce modèle transforme cartément l'activité économique du Mexique en une variable à la merci des investissements étrangers et de la disponibilité de fonds extérieurs. La stratégie économique est ainsi intimement liée à la libéralisation de l'activité économique, à l'ouverture commerciale et à une plus grande participation des intérêts étrangers dans l'économie mexicaine. Par conséquent, dès que ces flux de capitaux désertent le Mexique, quelle

7 Voir l'encadré ci-contre. 
qu'en soit la raison - qu'il s'agisse d'une hausse des taux d'intérêts fixés par la Réserve fédérale ou d'un événement politique négatif survenant au Mexique - l'économie nationale s'en ressent parce qu'elle s'est assujettie à ces flux de capitaux et qu'elle ne peut donc financer par elle-même l'énorme déficit en comptes courants qu'elle génère.

La Banque mondiale et le FMI, dont les politiques d'ajustement structurel sont nettement hostiles aux travailleurs, dénient toute responsabilité face aux désastreuses conséquences de leurs politiques. Dans le cas du Mexique, ils affirment sans rougir que ce sont les fonctionnaires du gouvernement mexicain qui se sont trompés en appliquant mal leurs recommandations ou encore qu'on aurait caché des informations aux experts de ces organismes. Par conséquent, le remède que ces deux institutions prescrivent au malade est toujours le même : ne pas laisser l'ajustement relâcher, mais, au contraire, le renforcer et le rendre permanent. L'effet que ce type de politiques a produit à la grandeur de l'Amérique latine a été le même partout : il n'a fait qu'exacerber les inégalités sociales.

Le $1^{\text {er }}$ janvier 1994, l'ALÉNA entrait en vigueur et, à peine trois mois et demi plus tard, le Mexique était admis comme membre à part entière de l'OCDE. Cet organisme, formé de 25 pays développés, étudie et évalue les programmes destinés à faciliter les processus d'un développement économique sain et l'expansion du commerce mondial. Un des premiers gestes du Mexique après son adhésion, en 1991, a été d'obtenir le statut d'observateur au Comité sur le travail et l'emploi, s'engageant par le fait même à suivre ses normes.

Le processus de mondialisation de l'économie mexicaine a, bien sûr, commencé il y a déjà plusieurs années avec la libéralisation des marchés stimulée par l'adhésion du pays au GATT, en 1986, et encore renforcée avec l'ALÉNA. Cette libéralisation se traduit par une pression additionnelle sur l'emploi, car elle permet aux entreprises transnationales d'exercer du chantage au niveau local sur les travailleurs de chaque pays. Ainsi, elles peuvent menacer d'abandonner le pays si les syndicats se mobilisent pour chercher à obtenir de meilleurs salaires, étant donné que leur implantation au niveau continental ou même à l'échelle planétaire leur permet de se déplacer librement. Le capital et les flux d'investissements ne rencontrent aucune barrière à leur mobilité alors que les travailleurs ne peuvent s'organiser à une échelle qui dépasse leurs frontières nationales.

Dans le cadre de l'ALÉNA, deux accords supplémentaires ont été "proposés" par l'administration Clinton et adoptés à contrecœur par le gouvernement mexicain: il s'agit de l'Accord nord-américain pour la 
coopération sur le travail et l'Accord nord-américain pour la coopération sur l'environnement. Dans ces deux accords parallèles, il n'y a que celui sur l'environnement qui permette un certain droit de regard aux citoyens.

L'administration Salinas a refusé d'inclure les droits du travail dans l'accord parallèle de l'ALÉNA. Comme on le sait, cet accord concerne uniquement le salaire minimum, le travail des enfants et la sécurité au travail. Des citoyens et des groupes de travailleurs tant au Mexique qu'aux États-Unis ont commencé à revenir sur le sujet auprès des Bureaux d'administration nationale de chacun des pays pour déposer des plaintes contre les entreprises qui violent les dispositions adoptées dans l'accord parallèle sur le travail, mais leurs actions ont été presque totalement inefficaces en raison surtout du manque de coopération des gouvernements 8 .

La coopération entre les travailleurs est devenue plus difficile en ces temps où les économies du Mexique, des États-Unis et du Canada se portent mal. Le sentiment anti-travailleur migrant, maintenant très répandu aux États-Unis, en fait foi, surtout dans les États du sud-ouest quand il s'agit des travailleurs mexicains. L'un d'eux disait : « nous sommes la principale exportation du Mexique et c'est peut-être pour cela que le gouvernement mexicain nous traite comme des marchandises... Il est davantage préoccupé par l'exportation de la bière Corona que de nous 9 \%.

Les salaires au Mexique sont une variable dépendante, soumise avant tout aux objectifs de promotion des exportations, d'attraction des investissements étrangers et de contrôle de l'inflation. Étant donné que l'ALÉNA a précisément pour but d'attirer des investissements étrangers et d'augmenter les exportations, il ne va qu'aggraver ce problème. Contrairement à d'autres pays, comme l'Allemagne ou le Japon, où la compétitivité des produits d'exportation repose sur l'éducation et la formation des travailleurs, dans le cas du Mexique, on a opté pour une compression drastique des salaires, recourant ainsi à une formule qui n'a évidemment pas fonctionné et qui n'a aucune chance de succès. Dans le cadre de cette politique punitive de l'effort des travailleurs, il n'y a pas d'incitation pour qu'ils augmentent leurs efforts et, par voie de conséquence, pour qu'ils

8 Parmi les causes les plus tristement célèbres, citons les plaintes enregistrées contre Honeywell, General Electric et Sony. Pour une documentation complète sur ces cas, contacter The International Labor Rights Education and Research Fund (ILRERF) à Washington, D.C.

9 Cité par Fausto Fernández PONTE, « Asimetrías », El Financiero, México, 7 novembre 1994. 
élèvent la productivité de leur travail. Le paradoxe, c'est que les registres des organismes officiels font valoir une augmentation de la productivité du secteur manufacturier au cours des dernières années, mais on arrive à ces résultats uniquement parce que la formule qui a servi à mesurer (volume de la production divisé par le nombre des travailleurs) a été faussée par les licenciements massifs dans le secteur en question.

Il faut encore ajouter que, dans le cas du Mexique, l'ouverture des frontières aux produits et services étrangers, une pièce maîtresse de la politique d'ajustement, a conduit à la désindustrialisation du pays. Comme plusieurs entreprises locales n'arrivent plus à être "compétitives", elles voient disparaître leur part de marché au profit de firmes étrangères et se font écarter du marché en raison de leurs prix qu'elles ne peuvent baisser suffisamment. Il est évident qu'un accroissement de la productivité du secteur manufacturier dans l'économie mexicaine devrait être poursuivi par le moyen d'une politique d'ensemble qui établisse des priorités (une véritable politique industrielle qui mette en synergie les ressources propres du Mexique, des programmes de formation des travailleurs, et par-dessus tout, des salaires décents), plutôt que de se contenter d'augmenter le nombre de producteurs inefficaces par le biais de la libéralisation des marchés.

Enfin, c'est seulement lorsque le processus de démocratisation atteindra le mouvement ouvrier que les travailleurs mexicains auront une possibilité de mieux contribuer à un développement juste et soutenu. Le Mexique ne deviendra pas un pays moderne sans qu'une pleine réforme démocratique n'y ouvre l'espace politique rendant possibles des mesures qui permettront aux travailleurs de profiter d'un processus de mondialisation qui a, jusqu'à présent, été monopolisé par une minorité aux échelons les plus élevés de la société. 